\title{
Stress-induced neuroinflammation: mechanisms and new pharmacological targets
}

\author{
C.D. Munhoz ${ }^{1}$, B. García-Bueno르, J.L.M. Madrigal², L.B. Lepsch ${ }^{1}$, C. Scavone ${ }^{1}$ and \\ J.C. Leza² \\ 1Departamento de Farmacologia, Instituto de Ciências Biomédicas, Universidade de São Paulo, \\ São Paulo, SP, Brasil \\ ${ }^{2}$ Departamento de Farmacologia, Facultad de Medicina, Universidad Complutense de Madrid and \\ CIBERSAM, Madrid, Spain
}

Correspondence to: J.C. Leza, Departamento de Farmacologia, Facultad de Medicina, Universidad Complutense de Madrid, 28040 Madrid, Spain. E-mail: jcleza@med.ucm.es

Stress is triggered by numerous unexpected environmental, social or pathological stimuli occurring during the life of animals, including humans, which determine changes in all of their systems. Although acute stress is essential for survival, chronic, longlasting stress can be detrimental. In this review, we present data supporting the hypothesis that stress-related events are characterized by modifications of oxidative/nitrosative pathways in the brain in response to the activation of inflammatory mediators. Recent findings indicate a key role for nitric oxide (NO) and an excess of pro-oxidants in various brain areas as responsible for both neuronal functional impairment and structural damage. Similarly, cyclooxygenase-2 (COX-2), another known source of oxidants, may account for stress-induced brain damage. Interestingly, some of the COX-2-derived mediators, such as the prostaglandin $15 \mathrm{~d}-\mathrm{PGJ}_{2}$ and its peroxisome proliferator-activated nuclear receptor PPAR $\gamma$, are activated in the brain in response to stress, constituting a possible endogenous anti-inflammatory mechanism of defense against excessive inflammation. The stress-induced activation of both biochemical pathways depends on the activation of the $N$-methyl-Daspartate (NMDA) glutamate receptor and on the activation of the transcription factor nuclear factor kappa $B(N F \kappa)$. In the case of inducible NO synthase (iNOS), release of the cytokine TNF- $\alpha$ also accounts for its expression. Different pharmacological strategies directed towards different sites in iNOS or COX-2 pathways have been shown to be neuroprotective in stress-induced brain damage: NMDA receptor blockers, inhibitors of TNF- $\alpha$ activation and release, inhibitors of NFKB, specific inhibitors of iNOS and COX-2 activities and PPAR $\gamma$ agonists. This article reviews recent contributions to this area addressing possible new pharmacological targets for the treatment of stress-induced neuropsychiatric disorders.

Key words: Cyclooxygenase; Nitric oxide; PPAR $\gamma$; Stress-induced neurodegeneration

Research supported by the Spanish Ministries of Health (\#ISCIII 01/0650, CIBERSAM to J.C. Leza) and Education (\#SAF0763138), and FAPESP (\#02/02298-2 to C. Scavone). C. Scavone is the recipient of a fellowship from CNPq (\#300097/2005-0), and C.D. Munhoz is the recipient of a fellowship from FAPESP (\#04/11042-7).

Received February 26, 2008. Accepted November 12, 2008

\section{Introduction}

The response of an organism to stress includes both physical and behavioral adaptations, as, for example, the "fight or flight" reaction. This response, also called hyperarousal or the acute stress response, was first described by Walter Cannon in 1915 (1) as the reaction to a threat with a general discharge of the sympathetic nervous system, priming the animal for fighting or fleeing. Some years later, the fight or flight response was recognized as the first stage of the general adaptation syndrome that regulates stress responses among vertebrates and other organisms. This syndrome was described by Selye (2) 70 years ago as a three-phase mechanism (alarm, adaptation 
and exhaustion) and was defined as the result of adaptation to situations in which an organism has to fight or flight to survive. Some of the physiological effects observed during the first phase of alarm correspond to a fast increase in catecholamines, a slow but sustained increase of glucocorticoids in the periphery and to a very fast increase in excitatory amino acids in many brain areas (3). These changes are essential for surviving acute physical stress, but they may cause adverse effects when secretion is sustained (4). Indeed, very intense or long-lasting stress results in a new biological equilibrium that can be either beneficial (e.g., exercise-induced conditioning of the cardiovascular system) or detrimental, causing damage or disease because of a maladaptation or allostasis (5).

The stress response affects many different organs and systems, mainly cardiovascular and central nervous system (CNS). The response of the cardiovascular system to stress has been ascribed mainly to catecholamine hyper-stimulation and involves increased cardiac output and vascular resistance, lipid mobilization, and stimulation of platelet aggregation. When exaggerated, these effects can contribute to the pathogenesis of hypertension, atherosclerosis, and ischemic heart disease (6). In the CNS of rats, stress causes reversible atrophy of hippocampal dendrites over the course of weeks (7) and initiates apoptotic changes in cortical and hippocampal areas (8). Furthermore, exposure to chronic unpredictable stress can cause permanent loss of neurons in rodents (7) and potentiated lipopolysaccharide (LPS)induced nuclear factor kappa $\mathrm{B}\left(\mathrm{NF}_{\kappa} \mathrm{B}\right)$ activation and $\mathrm{mRNA}$ expression of pro-inflammatory genes in the frontal cortex and hippocampus (9). In humans, atrophy of the hippocampus has been observed. It was first described in war-related post-traumatic stress disorder and major depression, coexisting with a decrease in neuronal activity (10). This atrophy, which also affects other areas (temporal lobe, orbitofrontal cortex) and whole brain volume, coexists with decreased blood flow and metabolism in prefrontal cortex of patients suffering depression (10).

Although stress is not a disease by itself, continuous exposure to stressful stimuli has been directly related to the onset, progression or outcome of many pathological processes. Some of the detrimental actions of stress hormones in the brain may be mediated via the release of secondary mediators. We will review the evidence on brain oxidative and nitrosative mediators in stress-induced injury and the basis for a possible pharmacological intervention.

\section{Physiology of stress}

The hypothalamus-pituitary-adrenal axis

The activation of mechanisms in response to stress is coordinated by the hypothalamus-pituitary-adrenal (HPA) axis. Although the pathways through which the brain translates the stressor nature of a particular stimulus into the integrated response at the hypothalamus are not known, neural inputs from catecholaminergic, serotonergic and cholinergic brain nuclei have been involved (11).

The parvocellular neurons of the paraventricular nucleus in the hypothalamus express corticotrophin-releasing factor. After experiencing a stress stimulus, the cells in the paraventricular nucleus secrete corticotrophin-releasing hormone. Once there, this substance acts on the anterior pituitary stimulating the proopiomelanocortin-producing cells to release adrenocorticotrophin. Adrenocorticotrophin is then released into the systemic circulation and transported to the adrenal cortical receptors where it induces the production of catecholamines and glucocorticoids. The detection of increased levels of these substances as well as sustained elevated levels of glucocorticoids in plasma is a typical feature of the stress response.

\section{Glucocorticoids}

Glucocorticoids are considered to be anti-inflammatory, immunosuppressive and immunomodulatory under normal conditions. The mechanisms underlying these effects of glucocorticoids in the periphery are well understood and include inhibition of lymphocyte proliferation and dendritic cell maturation, and apoptosis of basophils, eosinophils and T-cells (12). However, in recent years, the classic view that glucocorticoids are universally anti-inflammatory has been challenged at a variety of levels, mainly in the CNS. Indeed, in the brain, glucocorticoids are not uniformly anti-inflammatory, and can even be proinflammatory at the level of cell extravasation and migration as well as inflammatory messenger, and transcription factor levels (12).

Chronic and sustained release of glucocorticoids participates in the atrophy observed in certain brain areas after intense and long-lasting stressing situations (4). In fact, similar alterations are detected in patients suffering Cushing's syndrome, who have a chronic hyper-production of glucocorticoids. The excess of glucocorticoids damages the hippocampus and this can contribute to an even larger increase in the production of glucocorticoids because the feedback mechanism exerted by them on the HPA axis is inhibited (10).

One of the mechanisms proposed for this neurodegeneration due to glucocorticoid hyper-secretion is the inhibition of glucose transport in brain neurons and glia, which could be the result of the redirection of the energy towards the muscles in order to facilitate the escape from the stressor. These effects seem to be mediated by the GR 
(type II receptor of glucocorticoids) and are due to a translocation of the glucose transporter molecules from the cellular surface to inside the cell as well as a reduction in the levels of the glucose transporter mRNA (7).

Glucocorticoids also promote a reduction of ATP levels during neurological insults (7), an effect which has also been observed in brain cortex from rats exposed to stress (13).

Another important mechanism for glucocorticoids or stress-induced brain damage is their effects on excitatory amino acids. When neurological survival is threatened by the release of large quantities of glutamate, glucocorticoids worsen this situation increasing calcium mobilization inside the cells, as well as interfering with glutamate reuptake. These effects reflect both direct and indirect consequences of glucocorticoids: by altering profiles of subunits of calcium channels or by inhibiting $\mathrm{Ca}^{2+}$ ATPase (reviewed in Ref. 7). Glucocorticoids also worsen degenerative events downstream of the $\mathrm{Ca}^{2+}$ excess (cytoskeletal proteolysis, microtubule abnormalities and accumulation of reactive oxygen specie). This final point is particularly interesting, giving the increasing emphasis on the role of reactive oxygen species in neuronal injury, which is detailed below.

\section{Mechanisms by which stress induces oxidative/nitrosative damage in the brain}

\section{Release of excitatory amino acids}

It is well known that one of the main effects of the stress response is the release of large quantities of excitatory amino acids, such as aspartate and glutamate, in different brain areas, an effect occurring rapidly after the onset of stress (20 min to $2 \mathrm{~h}$ ) (3).

Released glutamate can bind to different receptors; the main one being the $N$-methyl-D-aspartate (NMDA) subtype, whose activation causes the mobilization of free cytosolic calcium. Excess in the intracellular calcium concentration over-activates certain calcium-dependent enzymes resulting in the generation of oxygen radicals, protein misfolding and cytoskeletal damage (14), constituting the process known as excitotoxicity.

Glucocorticoids potentiate the damaging effect of calcium not only by inducing its influx to the cytosol but also by inhibiting its efflux via the $\mathrm{Ca}^{2+} / \mathrm{ATP}$ ase and the $\mathrm{Ca}^{2+} / \mathrm{Na}^{+}$ exchanger (7). The relationship between the glucocorticoid-mediated effects on glutamate and the consequent neuronal alterations has also been observed in experimental models of stress (15).

The increase in the extracellular concentration of glutamate can be achieved not only by releasing larger quanti- ties of this amino acid but also by a reduction in its clearance. If the clearance of glutamate is not sufficient to avoid the over-stimulation of its receptors, subsequent cellular damage can be promoted (16). Both neurons and astroglia are capable of transporting glutamate by a high-affinity, sodium-dependent transport. To date, high-affinity, sodium-dependent glutamate transporters have been cloned from rodent and human tissue, known as excitatory amino acid transporters (EAAT) 1 to 5 (17). Immunohistochemical studies have revealed that EAAT1 and EAAT2 are present primarily in astrocytes, while EAAT3 and EAAT4 are distributed in neuronal membranes, and EAAT5 is retinal.

The involvement of transporter regulation and its modification by stress can be evaluated by studying synaptosomal glutamate uptake and expression of the main transporters EAAT1, EAAT2 and EAAT3 after different experimental stress protocols. Indeed, stress causes a decrease in synaptosomal glutamate uptake, indicating a dysfunction of glutamate transporters at this level (18). Furthermore, glutamate transporter (EAAT2 and EAAT3) expression in brain cortical membranes is decreased after stress, possibly explaining the increase in basal serum glutamate release detected in stressed animals (19).

This poses the question what mechanism is responsible for the increased glutamate release after stress? In certain situations such as ischemia, the transport systems responsible to maintain low extracellular glutamate levels can be reversed causing the opposite effect due to the fall in ATP production, i.e., increased release. This fall in ATP has also been observed in the brain of stressed animals mediating, at least in part, the subsequent reversal of the glutamate uptake systems and increasing the release of glutamate (20).

\section{Release of cytokines in the brain during stress}

Although a considerable amount of evidence has shown that physical and psychological stress elevates plasma levels of several cytokines (i.e., tumor necrosis factor $\alpha$, TNF- $\alpha$; interleukin-1, IL-1) in animals and humans subjected to psychological stress, anxiety states and anorexia nervosa, the physiological significance of this elevation is not known (21). Although most cytokines present in the brain after stress come from the periphery, there are important cellular sources of these mediators throughout the brain, mainly endothelial cells and epithelial cells of the choroid plexus and ventricles as well as glia and neurons from dentate gyrus of the hippocampus and the anterior pituitary, anterior olfactory nucleus, thalamus, hypothalamus, amygdala, and cerebellum (reviewed in Ref. 22).

One of these cytokines, TNF- $\alpha$, is rapidly produced in 
the brain in response to tissue injury. TNF is a group of cytokines that consists of two forms, namely the $\alpha$ and $\beta$ forms, which are the products of two different genes. Many studies have been focused on the $\alpha$ form because its excessive production can cause tissue damage (23). TNF$\alpha$ levels are increased in the CNS after damage due to traumatic injury, ischemia, infections, or diseases that involve brain degeneration, playing an important role in the adaptive response to these conditions.

TNF- $\alpha$ is released in its soluble form from its membrane-bound precursor by a membrane-anchored zinc metalloproteinase, identified as a disintegrin and metalloproteinase (ADAM) called TNF- $\alpha$ convertase (TACE/ ADAM17) (24). TACE cleaves pro-TNF- $\alpha$ at the cell surface releasing the mature form from the cell membrane. Like other cytokines, TNF- $\alpha$ exerts its effects by binding first to specific surface receptors on target cells. There are two types of TNF receptors: the p55 and the p75 also called TNFR-1 and -2, respectively (25).

Either TACE expression or activity has been found to change in some neuroinflammatory conditions in which an increase in TNF- $\alpha$ levels has been described, such as stroke, multiple sclerosis or traumatic injury (26). We have shown that after exposures to only $30 \mathrm{~min}$ of restraint stress TACE enzymatic activity in the brain cortex is increased (27). The predictable consequence of TACE activation is a larger release of TNF- $\alpha$. In fact, this has been found in brain cortex samples obtained from stressed rats. While the activity of TACE was increased after half an hour of immobilization, the levels of soluble TNF- $\alpha$ were increased one hour after the onset of stress. The implication of TACE in this process was demonstrated by the reduction in the TNF- $\alpha$ levels in stressed animals treated with the preferred inhibitor of TACE activity BB1101 (27). TNF- $\alpha$ has been identified also as a main regulator of persistence of the oxidative changes after sustained, inescapable and predictable stress (28).

Trying to go further in the sequence of events initiated by stress exposure, we also investigated the possible influence of glutamate in the release of TNF- $\alpha$ by blocking the NMDA glutamate receptor with the specific non-competitive compound MK-801 (dizocilpine). Interestingly, NMDA blockade not only decreases stress-induced activity and expression of TACE, but also its constitutive expression, as well as TNF- $\alpha$ levels (27). Taken together, these results demonstrate a crucial role for glutamate in the initiation of a response that shares certain features with the inflammatory processes. These results highlight a direct central regulatory effect of stress-induced neuroinflammation to be added to the entry of cytokines from outside the brain.
Activation of transcription factors during stress: NFkB

$\mathrm{NF} \kappa \mathrm{B}$ is a transcription factor widely expressed in the CNS. It consists of homodimers or heterodimers of a family of structurally related proteins. The most commonly found type is the heterodimer composed of a p65 (RelA) and a p50 subunit. This dimer can also bind to a third protein called $I_{\kappa} B$, which inactivates NF $\mathrm{BB}$. The activation of this transcription factor comprises the sequential activation of different enzyme complexes such as NFkB-induced kinase (NIK), which, in turn, activates I $\mathrm{KB}$ kinase (IKK) that catalyzes the phosphorylation of two serines in $\mathrm{NF}_{\kappa} \mathrm{B}$. This constitutes the signal for the degradation of $I_{\kappa} B$. Once free of the inhibitory subunit, $N F \kappa B$ can enter the nucleus where it binds to specific $\kappa B$ DNA consensus sequences in the enhancer region of a variety of $\kappa B$-responsive genes, some of which are involved in oxidative-nitrosative damage (29).

Stress activates NF $\kappa B$ in brain cells as soon as $4 \mathrm{~h}$ after the onset of stress in rats (reviewed in Ref. 19) and potentiates the activity of this transcription factor in the frontal cortex and hippocampus of rats under endotoxic shock (induced by LPS) (9). This activation of NFKB after stress was confirmed in experiments with humans and transgenic mice subjected to psychological or immobilization stress (30).

Many stimuli such as viral and bacterial infection, glutamate, UV light, ionizing radiation, free radicals, and cytokines activate $N F \kappa B(29)$. One of these activators is TNF$\alpha$ (31). The implication of TACE and TNF- $\alpha$ in stressinduced activation of $\mathrm{NF} \kappa \mathrm{B}$ was confirmed after finding that the pharmacological inhibition of TACE also blocked the stress-induced activation of $N F_{\kappa B}$ (27).

Additionally, the essential role of NMDA activation as a trigger factor in this process is of particular interest, since blockade of this receptor decreases NFKB translocation induced by LPS (9), and also decreases stress-induced TACE activation and TNF- $\alpha$ release (19).

We will now examine the consequences of the activation of such a transcription factor, leading to the expression of several responsive genes such as those involved in oxidative/nitrosative products.

\section{NFKB-related oxidative/nitrosative products in stress}

Some of the genes responsive to $\mathrm{NF} \kappa \mathrm{B}$ are oxidative/ nitrosative sources. These include inducible nitric oxide synthase (iNOS, NOS-2) and cyclooxygenase-2 (COX-2).

iNOS. Nitric oxide (NO) is synthesized from L-arginine by the enzymes NO synthases: endothelial, neuronal and an isoform expressed during inflammatory reactions (iNOS). iNOS is an enzyme expressed after exposure of cells to several noxious agents such as cytokines or LPS (32). 
This NOS isoenzyme mediates cytotoxicity in many cell systems, mainly because of the oxidative/nitrosative effects produced by extensive and prolonged release of $\mathrm{NO}$, which finally lead to a production of other oxidant species such as peroxynitrite (33).

Evidence has been presented for the role of $\mathrm{NO}$ in some pathological processes in the CNS. Indeed, the excessive generation of $\mathrm{NO}$ has been demonstrated in epilepsy, hypoxic-ischemic damage and neurodegenerative disorders including Alzheimer's and Parkinson's diseases and Huntington's corea (32).

It has been reported that exposure to stress results in increased NO production in the brain and periphery. A similar transient stimulation of $\mathrm{NO}$ release also occurs in plasma of humans subjected to stress (reviewed in Ref. 19). This systemic release of NO may offset the vasoconstrictor and pro-aggregatory effects of stress hormones and mediators during the first stages of the stress response.

Immobilization stress increases brain iNOS activity and expression as soon as $6 \mathrm{~h}$ after the onset of stress (34), and longer periods of stress produce higher levels of iNOS expression and activity (reviewed in Ref. 19). The detrimental effects of the long-term stress induction of iNOS include accumulation of membrane aldehyde products (peroxidation products), disruption of blood brain barrier and mitochondrial impairment (19). Pharmacological data obtained using specific inhibitors of iNOS support the conclusion of the participation of iNOS in stress-induced damage. It is unclear whether NO per se is the effector molecule of neuronal damage. A persistent elevation of $\mathrm{NO}$ levels can result in generation of a potent oxidant, peroxynitrite (ONOO-) from superoxide and NO. Peroxynitrite is a tissue-damaging agent that acts by the initiation of lipid peroxidation, oxidation of sulfhydryl groups and nitrosation of tyrosine-containing molecules. The huge accumulation of $\mathrm{NO}$ and over-expression of iNOS observed in the brain during chronic stress probably leads to the production of reactive oxygen or nitrogen species such as peroxynitrite (19).

iNOS induction in stress depends on NFאB activation. This was supported by the use of an inhibitor of $N F \kappa B$ activation such as pyrrolidine dithiocarbamate, which decreased the activity and expression of iNOS in stressed animals (34).

Pharmacological experiments were carried out to study the possibility that cytokines and excitatory amino acids account for the stress-induced activation of iNOS. Indeed, specific blockers of the NMDA receptor of glutamate (MK801) or TACE inhibitors (BB1101) partially inhibit the stressinduced iNOS expression and activation (25).
COX-2. As is the case for NOS, there are different isoforms of COX, namely COX $-1,-2$ and -3 . COX-1 is constitutively expressed in almost all tissues. COX-2 is induced and expressed by cells that are involved in inflammation (e.g., macrophages, monocytes, synoviocytes) and is responsible for the synthesis of prostanoids involved in pathological processes (31). COX-3 has been recently discovered as an RNA splicing variant of COX-1.

COX-2 is one of the most important components of the inflammatory response, and its reaction products are responsible for cytotoxicity in models of inflammation. Although COX-2 is expressed constitutively in brain cells, its expression is up-regulated in several neurological diseases, such as stroke, Alzheimer's dementia and seizures (35).

The mechanisms by which COX-2 produces cell damage involve the biosynthesis of prostaglandins, which generates free oxygen radicals that can result in injury. On the other hand, prostaglandin itself also can be responsible for the damage by inducing glutamate release from astrocytes or apoptosis (reviewed in Ref. 36).

The link between the action of COX-2 and neurological damage is deduced from numerous studies in which inhibition of induced COX-2 activity (37) or deletion of its gene (38) result in neuroprotection.

Studies have shown an increase in COX-2 mRNA levels due to forced swim stress in mice (39). Recently, an increased expression and activity of COX-2 in brain cortex has been observed after a short exposure (4-6 h) to restraint stress in rats (19). Interestingly, specific pharmacological tools that inhibit COX-2 activity (NS-398) prevent the increase on membrane lipid peroxidation mediators and the depletion of the main tissular antioxidant (glutathione), suggesting that this COX isoform is involved in the accumulation of oxidative mediators found in the brain after stress. The activity and expression of COX-2, as in the case of iNOS, is mediated by glutamate and NFКB (19).

\section{A COX-2-dependent mechanism of inflammatory balance: deoxy-prostaglandins and PPARS}

All organisms have complex defense mechanisms that allow them to adapt to and survive stress. Two antiinflammatory mechanisms have received great interest in the literature during the last years: one is the counterbalancing vagal reflex after sepsis, hemorrhagic shock, ischemia and other inflammatory syndromes, which inhibits cytokine release and thereby prevents tissue injury and death (reviewed in Ref. 40), and the other is carried out by certain cyclopentenone prostaglandins derived from the differen- 
tial activation of COX isoforms by different physio-pathological stimuli. This latter mechanism is beginning to be considered as a possible local endogenous regulator of the inflammatory response in neurodegenerative conditions and has received considerable attention from the stress scientists.

The most studied anti-inflammatory prostaglandin is the prostaglandin 15-deoxy-prostaglandin (15d-PGJ ${ }_{2}$ ), a structural, non-enzymatic derivative from the prostaglan$\operatorname{din} D_{2}$. This prostaglandin is the endogenous ligand proposed for the gamma isoform of the subfamily of peroxisome proliferator-activated nuclear receptors, PPAR $\gamma$. These receptors have been thought to be directly involved in the regulation of the inflammatory response in several animal models of neuropathologies having a clear associated inflammatory component (41). PPAR $\gamma$ also possesses a great number of synthetic ligands such as the family of anti-diabetic drugs known as thiazolidinediones (TZDs), which include rosiglitazone, troglitazone, pioglitazone, and ciglitazone (42).

Kainu et al. (43) carried out the first study demonstrating the presence of PPAR $\gamma$ mRNA and protein in CNS cells. Later, other more detailed studies have demonstrated that PPAR $\gamma$ presents a high degree of expression in the rat cerebral frontal cortex, in all layers and mainly in the neurons of layer II, showing immunostaining in the nuclear and cytoplasmic compartments (44). Also, PPAR $\gamma$ expression has been detected in microglial cells and in astrocytes, making these cells potential targets for the PPAR $\gamma$ ligand anti-inflammatory activity in neurological diseases having an associated inflammatory component (45).

PPAR $\gamma$ and its ligands are master regulators of cerebral physiology and potential therapeutic targets for the treatment of several pathological conditions within the CNS. A large number of animal models of neuropathologies (ischemia, Alzheimer's disease, multiple sclerosis, Parkinson's disease) have been used to study the effects of treatments with PPAR $\gamma$ agonists (41). In addition, some studies already indicate the utility of these compounds to treat certain gliomas and astrocytomas and to control lymphocyte T proliferation (41). Currently, all this evidence from basic research has reached the clinical level: clinical trials are being carried out to determine the effectiveness and safety of the treatment with PPAR $\gamma$ agonists in Alzheimer's disease and cerebral ischemia (46). At the moment, the results are promising. Also, trials on progressive secondary multiple sclerosis have shown positive results. Treatments with PPAR $\gamma$ agonists have been demonstrated to be effective also in neuroinflammation models in in vivo studies of neuropathologies such as Alzheimer's disease, multiple sclerosis and Parkinson's disease (47).
This promising anti-inflammatory pathway is also strongly related to stress exposure. In fact, studies from our laboratory have shown that acute immobilization stress enhances both the production of $15 \mathrm{~d}-\mathrm{PGJ}_{2}$ and the expression of PPAR $\gamma$ in the cortex of the rat brain, as demonstrated by Western blot and immunohistochemical analyses. The staining was particularly evident in layers II to VI of the cortex of stressed animals, in neurons and astrocytes (48).

We have also demonstrated that both synthetic and natural PPAR $\gamma$ ligands prevent the inflammatory and oxidative/nitrosative consequences of stress exposure in the CNS of rats subjected to immobilization stress (49). The mechanisms by which these compounds prevent these effects include inhibition of stress (acute and chronic)induced increase in iNOS activity, NFKB blockade (by preventing stress-induced $\mathrm{lkB} \alpha$ decrease) and inhibition of TNF- $\alpha$ release in stressed animals. Other research has indicated the capacity of the PPAR $\gamma$ agonists (TZDs and $15 \mathrm{~d}-\mathrm{PGJ}_{2}$ ) to reduce the expression of the pro-inflammatory cytokine TNF- $\alpha$ and iNOS, gelatinase B (MMP-9) and COX-2 in LPS-stimulated macrophages in glial cells and neurons. These proteins contribute to the inflammatory damage observed in certain neurological diseases (47). PPAR $\gamma$ agonists may also activate antioxidant pathways such as nuclear factor (erythroid-derived 2)-like 2 (Nrf2) (50).

The neuroprotection afforded by treatment with PPAR $\gamma$ agonists is extended to sub-acute restraint stress paradigms (7 or 14 consecutive days). In this way, treatment with PPAR $\gamma$ agonists exerts direct protective action on the cerebral glucose and glutamate metabolism disrupted after stress exposure (regulating the expression of the neuronal glucose transporter GLUT-3 and the predominant glial glutamate transporter EAAT-2), mechanisms to add to its above-discussed anti-inflammatory/antioxidant effects, adding new therapeutic implications to the management of patients at risk of stressful events (51).

All of these findings clearly support the view that immobilization stress activates PPAR $\gamma$ in the brain, and pharmacological manipulation of intrinsic pathways such as PPAR $\gamma$ could provide protection in neuroinflammatory conditions. A question of further interest is the possibility that this pathway serves as a mediator of "central neurogenic neuroprotection" or "adaptive plasticity", as proposed in the stressed brain. It has been recently demonstrated that this pathway is finely regulated in the brain by stress hormones, including catecholamines, glucocorticoids and excitatory amino acids (52).

The possible use of exogenously administered $P \mathrm{PJ}_{2}$ as a tool to maintain antiinflammatory balance in this 
condition deserves special attention. In fact, expression of COX-2 can be up- or down-regulated in situations of neuroinflammation. In basal conditions (inactivated stage), physiological $\mathrm{PGD}_{2}$ metabolites such as $\mathrm{PGJ}_{2}$ closes a negative feedback loop on COX-2 expression, whereas in activated stages, such as stress, COX-2 is activated by enhanced levels of $\mathrm{PGD}_{2}$ metabolites, with production of its main product, $\mathrm{PGE}_{2}$. By administering supraphysiological doses of $P \mathrm{FJ}_{2}$, as we do in stress, one could expect an inhibition in COX-2 expression and $\mathrm{PGE}_{2}$ production, as seen in different models (53). Accordingly, exogenous administration of $P G J_{2}$ should exert a more powerful antiinflammatory effect, as it occurs in the case of the antipyretic effect of this endogenous ligand. Thus, the antiinflammatory effect of $P G J_{2}$ is more apparent in stressful situations than in resting status, due to induction of endogenous $\mathrm{PGJ}_{2}$ production, which, when combined with exogenous $15 d-P G J_{2}$, could exert a more powerful effect (53). These observations support the rationale of using supraphysiological doses of $\mathrm{PGJ}_{2}$.

\section{Stress, aging and neurodegenerative diseases}

Stress, inflammation and NO have been shown to be involved in neurodegeneration. In fact, stress causes deficits especially in spatial memory performance, and this effect may be important for pathophysiological processes connected with aging, as well as degenerative diseases such as Alzheimer's and Parkinson's diseases (54). In addition, hippocampal atrophy may be involved and the stress-related activation of the CNS and/or HPA axis could represent a pathophysiological starting point (7). Moreover, the aging hippocampus apparently is more susceptible to stress, and this vulnerability may be increased in Alzheimer's disease (55). Stress has been demonstrated to be part of mechanisms related to anxiety and depression. NO may also be involved in the pathophysiology of anxiety/depression, since it interferes with various components and underlying mechanisms of the stress response (55). Studies performed on an animal model for chronic mild stress (a situation that mimics human depression) showed that the hippocampal atrophy of neurons present in these rats was inhibited by fluoxetine, an antidepressant that is capable of blocking NO production (56). Also, a recent study showed that the cytokine $\mathrm{IL}-1 \alpha$ is a critical mediator of the antineurogenic and depressive-like behavior caused by acute and chronic stress and this effect is mediated by the $\mathrm{NF}_{\kappa} \mathrm{B}$ signaling pathway (57).

Energy deficiency and dysfunction of $\mathrm{Na}$,K-ATPase are common consequences of many pathological insults and stress. Glutamate through cyclic guanosine mono- phosphate (GMP) and cyclic GMP-dependent protein kinase (PKG) has been shown to stimulate $\alpha_{2 / 3}-\mathrm{Na}$,K-ATPase activity in the CNS (58). Thus, a slight impairment of this pathway may amplify the disruption of ion homeostasis in the presence of a non-lethal insult. Studies in rats suggest that basal age-related decline in sodium pump activity is a consequence of changes in different steps of the cyclic GMP-PKG pathway. On the other hand, age-related reduction in glutamate-positive modulation of cerebellar $\alpha_{2 / 3^{-}}$ $\mathrm{Na}, \mathrm{K}-\mathrm{ATP}$ ase is linked to a defective PKG signaling pathway (59). The loss of the ability of $\alpha_{2 / 3}-\mathrm{Na}, \mathrm{K}-\mathrm{ATP}$ ase to respond to glutamate through a cyclic GMP-PKG cascade could be a failure in an important mechanism for rectifying ionic disturbances that may be present in aging processes and may predispose to or potentiate an effect of stress in the manifestation of age-related degenerative disorders. In fact, chronic predictable and unpredictable stress decrease the neuronal $\mathrm{Na}, \mathrm{K}-\mathrm{ATP}$ ase activity and high levels of glucocorticoid have been detected in 24-month-old rats (Munhoz CD, Scavone C, unpublished results), which could induce a further reduction of ATP levels during a neurological insult. It is interesting to note that the aging process (30-month-old animals) induces up-regulation in constitutive NFKB binding activity in the frontal cortex, which in the presence of glucocorticoid levels could potentiate LPS-induced NFKB activation and an increase in mRNA of proinflammatory genes (9). In addition, rats with increased HPA reactivity induced by prenatal stress or by the absence of neonatal handling show an early decline of cognitive functions associated with the hippocampus, as well as increased propensity to self-administer drugs such as amphetamine and cocaine (55). In addition, exposure to both chronic restraint and unpredictable stress increased cocaine-induced locomotion and basal corticosterone plasma levels and chronic unpredictable stress also displayed the largest locomotor response following a challenge dose with cocaine compared with control and chronic restraint stress groups (60). Drug abuse is associated with changes in brain function and neurodegenerative processes, which, for some drugs, have been shown to be associated with the induction of apoptotic/necrotic cell death (Lepsch LB, Munhoz CD, Kawamoto EM, Lima LS, Scavone C, unpublished results). Thus, life-long patterns of HPA function are probably important to determine the susceptibility of the body to stress or insults during the aging process.

\section{Future directions}

The impact of stressor exposure on brain function has been widely investigated in the last years and is a main concern of current neuropsychiatry. Furthermore, the num- 
ber of reports indicating that long-lasting stress affects synaptic plasticity, dendritic morphology and neurogenesis in animals and induces both clinical and anatomical features of neurotoxic damage in humans (i.e., post-traumatic stress disorder) is increasing. The vulnerability of many systems of the body to stress in aging is therefore

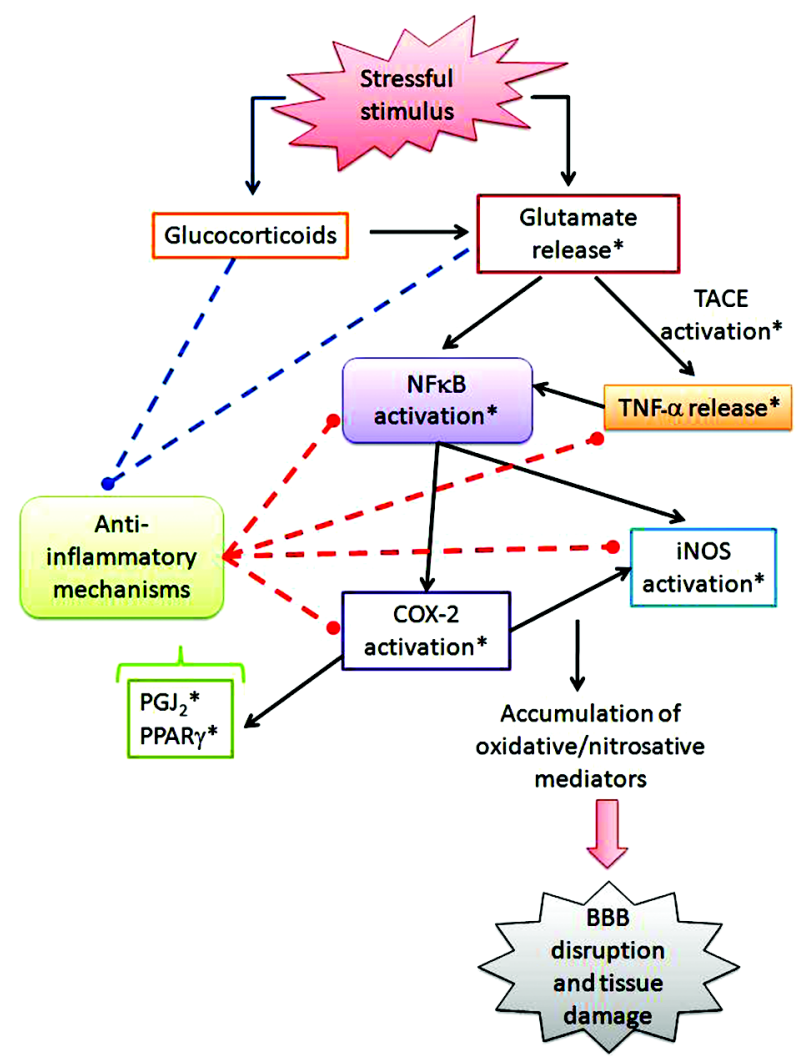

Figure 1. Stress-induced oxidative/nitrosative mediators in the brain. TACE $=$ TNF $-\alpha$ convertase; NF $\kappa B=$ nuclear factor kappa $\mathrm{B}$; TNF- $\alpha=$ tumor necrosis factor alpha; iNOS = inducible nitric oxide synthase; $C O X-2$ = cyclooxygenase- 2 ; $P \mathrm{PJ}_{2}=$ prostaglandin $15 \mathrm{~d}-\mathrm{PGJ}_{2}$; PPAR $\gamma=$ peroxisome proliferator-activated nuclear receptor gamma; $\mathrm{BBB}=$ blood-brain barrier. ${ }^{*}$ Possible targets for drugs. Straight line $=$ increase; dotted line $=$ decrease . influenced by experiences during life and is probably linked to the reactivity of the HPA axis. The main clinical correlates of stress-related neuropsychiatric disorders are depressive disorders, which are among the most common neuropsychiatric illnesses and represent a significant public health problem. The precise molecular and cellular events induced by stress and responsible for the brain damage found in this condition are still a matter of debate, but recent investigations point out a role for oxidativenitrosative mediators.

The high-output isoform of iNOS (NOS-2) has been implicated in cellular toxicity in many cell systems including brain. In this context, it has been demonstrated that stress induces the expression of iNOS in rat brain and that its inhibition protects against stress-induced cell damage in this model. Once iNOS is expressed, the formation of large amounts of oxygen and nitrogen reactive species may account for the oxidation of cellular components found after stress in the rat brain. A plausible consequence of this is the volume reduction that has been described in several brain structures in chronically depressed patients and other stress-related disorders.

On the other hand, the COX pathway has also been implicated in stress-induced brain damage. COX-2 is induced in stress and has been involved in the damage associated with this condition. According to its condition of inducible enzyme, and similarly to iNOS, the promoter of the immediate-early gene COX-2 depends on the activation of $\mathrm{NF} \kappa \mathrm{B}$ in stress. Both enzymatic sources of oxidative mediators in the brain depend on the activation of the NMDA type of glutamate receptor, and, in the case of iNOS, its activation also depends on the release of TNF- $\alpha$.

The findings reviewed here indicate that excitatory amino acids and subsequent activation of $N F \kappa B$ account for stress-induced iNOS and COX-2 expression and activity in the cerebral cortex (Figure 1). They support a possible neuroprotective role for specific inhibitors in this situation. The usefulness of PPAR agonists and $P G J_{2}$ as a tool for down-regulation of brain inflammation is an important area to be developed in the future.

\section{References}

1. Cannon WB. Bodily changes in pain, hunger, fear and rage: An account of recent researches into the function of emotional excitement. New York: Appleton; 1915.

2. Selye H. A syndrome produced by diverse nocuous agents. Nature 1936; 138: 32.

3. Moghaddam B. Stress preferentially increases extraneuronal levels of excitatory amino acids in the prefrontal cortex: comparison to hippocampus and basal ganglia. $J$ Neurochem 1993; 60: 1650-1657.

4. Sapolsky RM. Why stress is bad for your brain. Science 1996; 273: 749-750.

5. McEwen BS. Protective and damaging effects of stress mediators. N Engl J Med 1998; 338: 171-179.

6. Esch T, Stefano GB, Fricchione GL, Benson H. Stress in 
cardiovascular diseases. Med Sci Monit 2002; 8: RA93RA101.

7. McEwen BS, Sapolsky RM. Stress and cognitive function. Curr Opin Neurobiol 1995; 5: 205-216.

8. Lucassen PJ, Vollmann-Honsdorf GK, Gleisberg M, Czeh B, De Kloet ER, Fuchs E. Chronic psychosocial stress differentially affects apoptosis in hippocampal subregions and cortex of the adult tree shrew. Eur J Neurosci 2001; 14: $161-166$

9. Munhoz CD, Lepsch LB, Kawamoto EM, Malta MB, Lima LS, Avellar MC, et al. Chronic unpredictable stress exacerbates lipopolysaccharide-induced activation of nuclear factor-kappaB in the frontal cortex and hippocampus via glucocorticoid secretion. J Neurosci 2006; 26: 3813-3820.

10. Bremner JD, Vythilingam M, Vermetten E, Nazeer A, Adil J, Khan S, et al. Reduced volume of orbitofrontal cortex in major depression. Biol Psychiatry 2002; 51: 273-279.

11. Frohman LA. Clinical neuropharmacology of hypothalamic releasing factors. N Engl J Med 1972; 286: 1391-1397.

12. Sorrells SF, Sapolsky RM. An inflammatory review of glucocorticoid actions in the CNS. Brain Behav Immun 2007; 21: 259-272.

13. De Cristobal J, Madrigal JL, Lizasoain I, Lorenzo P, Leza $\mathrm{JC}$, Moro MA. Aspirin inhibits stress-induced increase in plasma glutamate, brain oxidative damage and ATP fall in rats. Neuroreport 2002; 13: 217-221.

14. Lipton P. Ischemic cell death in brain neurons. Physiol Rev 1999; 79: 1431-1568.

15. Magarinos AM, McEwen BS. Stress-induced atrophy of apical dendrites of hippocampal CA3c neurons: involvement of glucocorticoid secretion and excitatory amino acid receptors. Neuroscience 1995; 69: 89-98.

16. Rothstein JD, Dykes-Hoberg M, Pardo CA, Bristol LA, Jin L, Kuncl RW, et al. Knockout of glutamate transporters reveals a major role for astroglial transport in excitotoxicity and clearance of glutamate. Neuron 1996; 16: 675-686.

17. Beart PM, O'Shea RD. Transporters for L-glutamate: an update on their molecular pharmacology and pathological involvement. Br J Pharmacol 2007; 150: 5-17.

18. Leza JC, Salas E, Sawicki G, Russell JC, Radomski MW. The effects of stress on homeostasis in JCR-LA-cp rats: the role of nitric oxide. J Pharmacol Exp Ther 1998; 286: 13971403.

19. Madrigal JL, Garcia-Bueno B, Caso JR, Perez-Nievas BG, Leza JC. Stress-induced oxidative changes in brain. CNS Neurol Disord Drug Targets 2006; 5: 561-568.

20. Lawrence MS, Sapolsky RM. Glucocorticoids accelerate ATP loss following metabolic insults in cultured hippocampal neurons. Brain Res 1994; 646: 303-306.

21. Dunn AJ, Wang J, Ando T. Effects of cytokines on cerebral neurotransmission. Comparison with the effects of stress. Adv Exp Med Biol 1999; 461: 117-127.

22. Dantzer R, Kelley KW. Twenty years of research on cytokine-induced sickness behavior. Brain Behav Immun 2007; 21: 153-160.

23. Wang CX, Shuaib A. Involvement of inflammatory cytokines in central nervous system injury. Prog Neurobiol 2002; 67: 161-172.

24. Black RA, White JM. ADAMs: focus on the protease domain. Curr Opin Cell Biol 1998; 10: 654-659.

25. Smith RA, Baglioni C. Characterization of TNF receptors.
Immunol Ser 1992; 56: 131-147

26. Hurtado O, Lizasoain I, Fernandez-Tome P, AlvarezBarrientos A, Leza JC, Lorenzo P, et al. TACE/ADAM17TNF-alpha pathway in rat cortical cultures after exposure to oxygen-glucose deprivation or glutamate. J Cereb Blood Flow Metab 2002; 22: 576-585.

27. Madrigal JL, Hurtado O, Moro MA, Lizasoain I, Lorenzo P, Castrillo $A$, et al. The increase in TNF-alpha levels is implicated in NF-kappaB activation and inducible nitric oxide synthase expression in brain cortex after immobilization stress. Neuropsychopharmacology 2002; 26: 155-163.

28. Munhoz C, Madrigal JL, Garcia-Bueno B, Pradillo JM, Moro MA, Lizasoain I, et al. TNF-alpha accounts for short-term persistence of oxidative status in rat brain after two weeks of repeated stress. Eur J Neurosci 2004; 20: 1125-1130.

29. Baldwin AS Jr. The NF-kappa B and I kappa B proteins: new discoveries and insights. Annu Rev Immunol 1996; 14: 649683.

30. Bierhaus A, Wolf J, Andrassy M, Rohleder N, Humpert PM, Petrov $D$, et al. A mechanism converting psychosocial stress into mononuclear cell activation. Proc Natl Acad Sci U S A 2003; 100: 1920-1925.

31. Li N, Karin M. Is NF-kappaB the sensor of oxidative stress? FASEB J 1999; 13: 1137-1143.

32. Moncada S, Palmer RM, Higgs EA. Nitric oxide: physiology, pathophysiology, and pharmacology. Pharmacol Rev 1991; 43: 109-142.

33. Beckman JS, Koppenol WH. Nitric oxide, superoxide, and peroxynitrite: the good, the bad, and ugly. Am J Physiol 1996; 271: C1424-C1437.

34. Madrigal JL, Moro MA, Lizasoain I, Lorenzo P, Castrillo A, Bosca $L$, et al. Inducible nitric oxide synthase expression in brain cortex after acute restraint stress is regulated by nuclear factor kappaB-mediated mechanisms. J Neurochem 2001; 76: 532-538.

35. Nogawa S, Zhang F, Ross ME, ladecola C. Cyclo-oxygenase-2 gene expression in neurons contributes to ischemic brain damage. J Neurosci 1997; 17: 2746-2755.

36. Vesce S, Rossi D, Brambilla L, Volterra A. Glutamate release from astrocytes in physiological conditions and in neurodegenerative disorders characterized by neuroinflammation. Int Rev Neurobiol 2007; 82: 57-71.

37. Domoki F, Perciaccante JV, Puskar M, Bari F, Busija DW. Cyclooxygenase-2 inhibitor NS398 preserves neuronal function after hypoxia/ischemia in piglets. Neuroreport 2001; 12 : 4065-4068.

38. Iadecola C, Niwa K, Nogawa S, Zhao X, Nagayama M, Araki $\mathrm{E}$, et al. Reduced susceptibility to ischemic brain injury and $\mathrm{N}$-methyl-D-aspartate-mediated neurotoxicity in cyclooxygenase-2-deficient mice. Proc Natl Acad Sci U S A 2001; 98: 1294-1299.

39. Yamagata $\mathrm{K}$, Andreasson $\mathrm{KI}$, Kaufmann WE, Barnes CA, Worley PF. Expression of a mitogen-inducible cyclooxygenase in brain neurons: regulation by synaptic activity and glucocorticoids. Neuron 1993; 11: 371-386.

40. Tracey KJ. Physiology and immunology of the cholinergic antiinflammatory pathway. J Clin Invest 2007; 117: 289296.

41. Feinstein DL. Therapeutic potential of peroxisome proliferator-activated receptor agonists for neurological disease. Diabetes Technol Ther 2003; 5: 67-73. 
42. Lehmann JM, Moore LB, Smith-Oliver TA, Wilkison WO, Willson TM, Kliewer SA. An antidiabetic thiazolidinedione is a high affinity ligand for peroxisome proliferator-activated receptor gamma (PPAR gamma). J Biol Chem 1995; 270: 12953-12956.

43. Kainu T, Wikstrom AC, Gustafsson JA, Pelto-Huikko M. Localization of the peroxisome proliferator-activated receptor in the brain. Neuroreport 1994; 5: 2481-2485.

44. Moreno S, Farioli-Vecchioli S, Ceru MP. Immunolocalization of peroxisome proliferator-activated receptors and retinoid $X$ receptors in the adult rat CNS. Neuroscience 2004; 123 : 131-145.

45. Luna-Medina R, Cortes-Canteli M, Alonso M, Santos A, Martinez A, Perez-Castillo A. Regulation of inflammatory response in neural cells in vitro by thiadiazolidinones derivatives through peroxisome proliferator-activated receptor gamma activation. J Biol Chem 2005; 280: 21453-21462.

46. Sundararajan S, Jiang Q, Heneka M, Landreth G. PPARgamma as a therapeutic target in central nervous system diseases. Neurochem Int 2006; 49: 136-144.

47. Heneka MT, Landreth GE. PPARs in the brain. Biochim Biophys Acta 2007; 1771: 1031-1045.

48. Garcia-Bueno B, Madrigal JL, Lizasoain I, Moro MA, Lorenzo P, Leza JC. Peroxisome proliferator-activated receptor gamma activation decreases neuroinflammation in brain after stress in rats. Biol Psychiatry 2005; 57: 885-894.

49. Garcia-Bueno B, Madrigal JL, Lizasoain I, Moro MA, Lorenzo P, Leza JC. The anti-inflammatory prostaglandin 15d-PGJ2 decreases oxidative/nitrosative mediators in brain after acute stress in rats. Psychopharmacology 2005; 180: 513-522.

50. Park EY, Cho IJ, Kim SG. Transactivation of the PPARresponsive enhancer module in chemopreventive glutathione S-transferase gene by the peroxisome proliferator-activated receptor-gamma and retinoid $\mathrm{X}$ receptor heterodimer. Cancer Res 2004; 64: 3701-3713.

51. Garcia-Bueno B, Caso JR, Perez-Nievas BG, Lorenzo P, Leza JC. Effects of peroxisome proliferator-activated recep- tor gamma agonists on brain glucose and glutamate transporters after stress in rats. Neuropsychopharmacology 2007; 32: 1251-1260.

52. Garcia-Bueno B, Madrigal JL, Perez-Nievas BG, Leza JC. Stress mediators regulate brain prostaglandin synthesis and peroxisome proliferator-activated receptor-gamma activation after stress in rats. Endocrinology 2008; 149: 19691978.

53. Mouihate A, Boisse L, Pittman QJ. A novel antipyretic action of 15-deoxy-Delta12,14-prostaglandin $\mathrm{J} 2$ in the rat brain. J Neurosci 2004; 24: 1312-1318.

54. Smith AD, Castro SL, Zigmond MJ. Stress-induced Parkinson's disease: a working hypothesis. Physiol Behav 2002; 77: 527-531.

55. Esch T, Stefano GB, Fricchione GL, Benson H. The role of stress in neurodegenerative diseases and mental disorders. Neuro Endocrinol Lett 2002; 23: 199-208.

56. Luo L, Tan RX. Fluoxetine inhibits dendrite atrophy of hippocampal neurons by decreasing nitric oxide synthase expression in rat depression model. Acta Pharmacol Sin 2001; 22: 865-870.

57. Koo JW, Duman RS. IL-1beta is an essential mediator of the antineurogenic and anhedonic effects of stress. Proc Natl Acad Sci U S A 2008; 105: 751-756.

58. Munhoz CD, Kawamoto EM, de Sa Lima L, Lepsch LB, Glezer I, Marcourakis T, et al. Glutamate modulates sodium-potassium-ATPase through cyclic GMP and cyclic GMP-dependent protein kinase in rat striatum. Cell Biochem Funct 2005; 23: 115-123.

59. Scavone C, Munhoz CD, Kawamoto EM, Glezer I, de Sa Lima L, Marcourakis T, et al. Age-related changes in cyclic GMP and PKG-stimulated cerebellar Na,K-ATPase activity. Neurobiol Aging 2005; 26: 907-916.

60. Lepsch LB, Gonzalo LA, Magro FJ, Delucia R, Scavone C, Planeta CS. Exposure to chronic stress increases the locomotor response to cocaine and the basal levels of corticosterone in adolescent rats. Addict Biol 2005; 10: 251-256. 